\title{
II. Symposium of Young Researchers on Pharmaceutical Technology, Biotechnology and Regulatory Science
}

\author{
January $23-24^{\text {th }}$ 2020. Szeged, Hungary
}

OP-21

DOI: $10.14232 /$ syrptbrs.2020.op21

The effect of bovine serum albumin concentration on lyophilized formulation characteristics

\author{
Maja Bjelošević, Mirjam Gosenca Matjaž, Mirjana Gašperlin, Pegi Ahlin Grabnar* \\ University of Ljubljana, Faculty of Pharmacy, Chair of Pharmaceutical Technology, Aškerčeva c. 7, 1000 Ljubljana, \\ Slovenia
}

Biopharmaceuticals represent one of the crucial areas in the pharmacy and are widely used to treat several diseases. Protein molecules are typically unstable in aqueous media, thus lyophilisation is the method of choice to transform such formulations into solid state. Initially biopharmaceuticals were intended for intravenous administration, whereas current trends are oriented in development of protein formulations for subcutaneous administration, which is associated with many challenges [1].

The main aim was to evaluate the effect of bovine serum albumin (BSA) concentration on formulation viscosity, further on related to critical quality attributes of lyophilized formulations, such as reconstitution time and visual appearance.

Formulations with five different BSA concentrations $(5,20,50,70$ and $100 \mathrm{mg} / \mathrm{mL}$ ) in phosphate buffer and sucrose or sucrose/mannitol as excipients were subjected to lyophilisation. Lyophilised products were evaluated for visual appearance, reconstitution time and particle size by dynamic light scattering. In addition, viscosity and thermal characteristics of pre-lyophilized solutions were determined.

Obtained results revealed that by increasing BSA concentration the reconstitution time was prolonged, while cake appearance was improved. Independent of formulation composition, the particle diameter was below $9 \mathrm{~nm}$ indicating absence of aggregates. Furthermore, an increase of viscosity with increasing BSA concentration was demonstrated.

The study indicates that BSA concentration along with excipient added have a decisive impact on lyophilized formulation characteristics, namely prolonged reconstitution time and increased viscosity both represent a main challenge in development of highly concentrated protein formulations.

References

1. Garidel P et al. Eur. J Pharm. Biopharm. 119, 353-360 (2017)

Supervisor(s): Mirjam Gosenca Matjaž, Mirjana Gašperlin, Pegi Ahlin Grabnar 